\title{
Assessment of a Neural-Network-Based Optimization Tool: A Low Specific-Speed Impeller Application
}

\author{
Matteo Checcucci, ${ }^{1}$ Federica Sazzini, ${ }^{1}$ Michele Marconcini, ${ }^{1}$ Andrea Arnone, ${ }^{1}$ Mario Coneri, ${ }^{2}$ \\ Luigi De Franco, ${ }^{2}$ and Matteo Toselli ${ }^{2}$ \\ 1 "Sergio Stecco" Department of Energy Engineering, University of Florence, Via di Santa Marta 3, 50139 Firenze, Italy \\ ${ }^{2}$ Termomeccanica Pompe S.p.A, Via del Molo 3, 19126 La Spezia, Italy \\ Correspondence should be addressed to Michele Marconcini, michele.marconcini@arnone.de.unifi.it
}

Received 30 November 2010; Accepted 7 May 2011

Academic Editor: Ken Ichi Funazaki

Copyright (C) 2011 Matteo Checcucci et al. This is an open access article distributed under the Creative Commons Attribution License, which permits unrestricted use, distribution, and reproduction in any medium, provided the original work is properly cited.

\begin{abstract}
This work provides a detailed description of the fluid dynamic design of a low specific-speed industrial pump centrifugal impeller. The main goal is to guarantee a certain value of the specific-speed number at the design flow rate, while satisfying geometrical constraints and industrial feasibility. The design procedure relies on a modern optimization technique such as an ArtificialNeural-Network-based approach (ANN). The impeller geometry is parameterized in order to allow geometrical variations over a large design space. The computational framework suitable for pump optimization is based on a fully viscous three-dimensional numerical solver, used for the impeller analysis. The performance prediction of the pump has been obtained by coupling the CFD analysis with a 1D correlation tool, which accounts for the losses due to the other components not included in the CFD domain. Due to both manufacturing and geometrical constraints, two different optimized impellers with 3 and 5 blades have been developed, with the performance required in terms of efficiency and suction capability. The predicted performance of both configurations were compared with the measured head and efficiency characteristics.
\end{abstract}

\section{Introduction}

The optimum configuration of a centrifugal pump is a compromise between reliability, low-cost manufacturing and high aerodynamic efficiency (Gopalakrishnan [1]). Even if reliability is still the most important factor, producers are pushing the limits of efficiency to develop energy-saving pumps. Moreover, the competitivity of the business requires the design process to be as brief as possible. Nowadays, the exponential increase of computational power has made approaches based on automating the conventional design process possible, coupling an optimization method with a computational fluid dynamic code (e.g., Pierret and Van Den Braembussche [2], Ashihara and Goto [3], Goto et al. [4], Pierret et al. [5], Bonaiuti et al. [6,7], Casey et al. [8]). These methods lead to a design process that relies more on a systematic methodology than on experience. They are less time consuming than the traditional approaches, which require the designer to continuously refine the component geometry.
In this context, Termomeccanica Pompe S.p.A. and the "Sergio Stecco" Department of Energy Engineering at the University of Florence started a joint research project for the design of pump stages, mainly based on the use of CFD techniques coupled with modern optimization tools.

The work described in this paper deals with the fluid dynamic design and optimization of the centrifugal impeller of a low specific-speed pump. The optimization campaign aimed at matching the design point and the best efficiency point with a prescribed specific-speed number, together with an overall minimum value of the pump efficiency and a good suction capability.

The design of the impeller was carried out using an in-house parameterization tool (e.g., Bonaiuti et al. [9]), a three-dimensional CFD code, and a post-processing tool, based on correlations. Sobol's quasi-random sequence of data was used in order to obtain a sampling of the design space and the inputs to perform the calculations needed as a training set. Artificial Neural Network (ANN), a fast, reliable, 


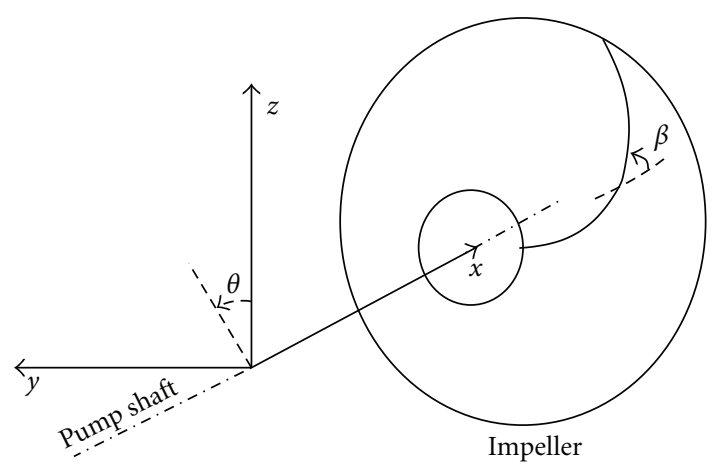

FIGURE 1: Cylindrical coordinate system.

and computationally inexpensive tool, was trained on the CFD results to obtain the surface response.

In order to obtain a prediction of the pump performance, the impeller was coupled with all the static components. These ones, especially the volute, are key factors for the performance of a low specific-speed centrifugal pump. Their flow environment is very complex, and the performance prediction is a challenging task for CFD. Moreover, a CFD simulation of the whole pump is an extremely timeconsuming task. For these reasons, a simple 1D model based on correlations was used to account for their influence on the pump. The proposed approach was validated against the experimental data available for an existing pump with a specific-speed number $N_{s}=10.9$ at its best efficiency point. The results were also considered representative of the new pump to be designed with a lower specific-speed number of $N_{s}=9.5$. In fact, in order to keep the manufacturing costs as low as possible, the requirement for the new pump is that all the other components, except the impeller itself, have to be the same as the pump with $N_{s}=10.9$.

Two different optimizations were performed aiming at obtaining a 3-blade and a 5-blade impeller, while satisfying geometrical constraints and industrial feasibility. A multistep approach was considered for the first one, while a global approach was used for the second one. After the optimization, both impellers were manufactured, and the predicted performance was verified against the experimental data in terms of head and efficiency characteristics.

\section{Computational Framework}

2.1. Blade Description and Parameterization. The threedimensional impeller geometry is handled in a parametric way. In a direct design procedure, it is essential to parameterize the component using as few geometrical parameters as possible. To this end, the geometrical properties of integral B-spline curves are exploited, for which the shape of the curve can be controlled using a few control points. A three-dimensional cylindrical coordinate system $((x, \theta, r)$ see Figure 1$)$ is oriented with the $x$-axis corresponding to the pump shaft and is used for blade description and parameterization.

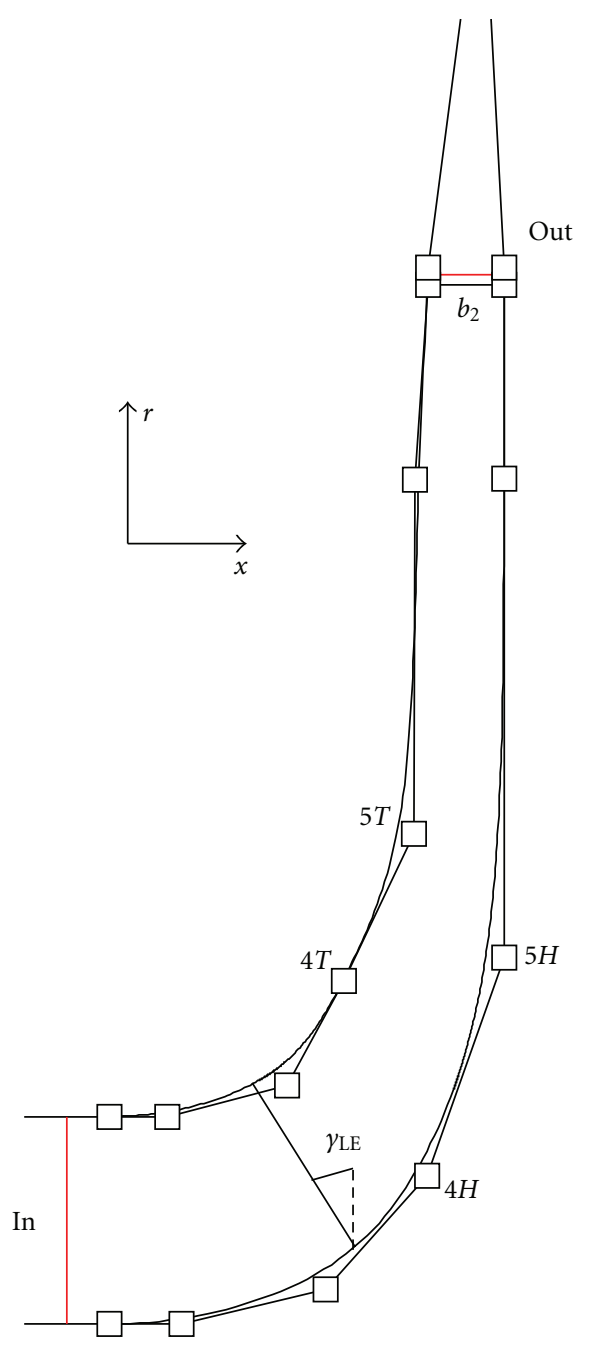

Figure 2: Meridional channel.

The input of the parameterization tool are the following geometrical data.

(i) Meridional Layout. Hub and shroud meridional contours are described in the $x-r$ plane by B-spline curves, while leading (LE) and trailing (TE) edges are described by a three-control-point Bèzier curve (Figure 2).

(ii) Blade Turning Angle $\beta_{b}$. The blade geometry is built by stacking in the $(m, r)$ plane a number of bladeto-blade sections. The camber line of each section is parameterized in terms of the turning angle $\beta_{b}$ along the meridional abscissa $m$. The $\beta_{b}$ angle, positive for counterclockwise rotation according to the coordinate system, is related to the azimuthal blade angle $\theta$ (see Figure 3 ) by

$$
\tan \beta_{b} \equiv r \frac{d \theta}{d m}
$$

the use of $\beta_{b}$ angle instead of $\theta$ allows the designer to easily control the blade load distribution. 


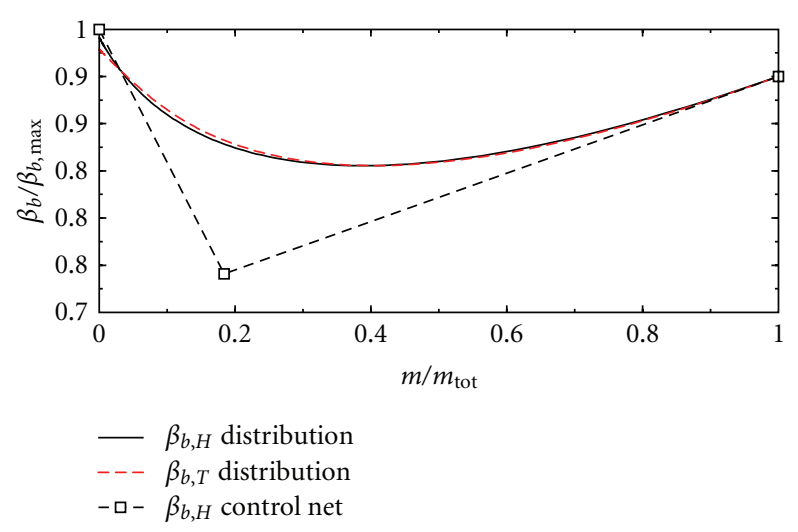

(a)

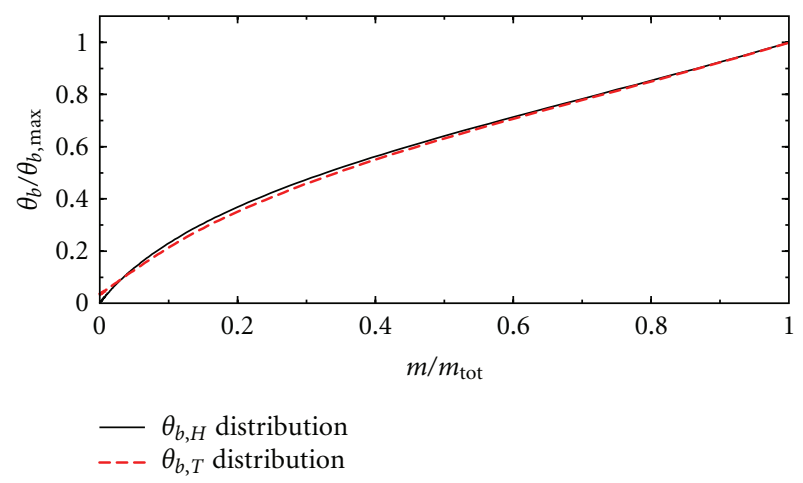

(b)

FIGURE 3: $\beta_{b}$ angle parameterization and corresponding $\theta_{b}$ angles at hub and tip.

(iii) Blade Thickness. A normal thickness distribution is assigned in order to define the blade profile.

(iv) Stacking Law. The stacking of the blade is determined by assigning the value of $\Delta \theta$ at a fixed $m$ for each section with respect to a reference one (usually the hub value).

2.2. Computational Grid. The impeller computational domain was discretized with a structured elliptic singleblock H-grid with $109 \times 73 \times 73$ points, for a total of 580.861 nodes. In order to guarantee a sufficient numerical resolution, the mesh was selected on the basis of a sensitivity analysis. Nonperiodic grids are employed in all computations presented here. The removal of periodicity allows the grid to reduce the mesh skewness, even for cascades having a large camber or a high stagger angle and twist. A pinch in the meridional channel, downstream of the impeller, was used in order to suppress regions of separated flow at the outlet section, which may affect the convergence and make it difficult to evaluate the component performance. Views of the $3 \mathrm{D}$ impeller and the $2 \mathrm{D}$ meridional channel grids are reported in Figures 4 and 5.

2.3. Flow Solver. The multirow, multiblock, incompressible single-phase version of the TRAFMS code (Arnone [10, 11], and Arnone and Pacciani [12]), a 3D solver originally

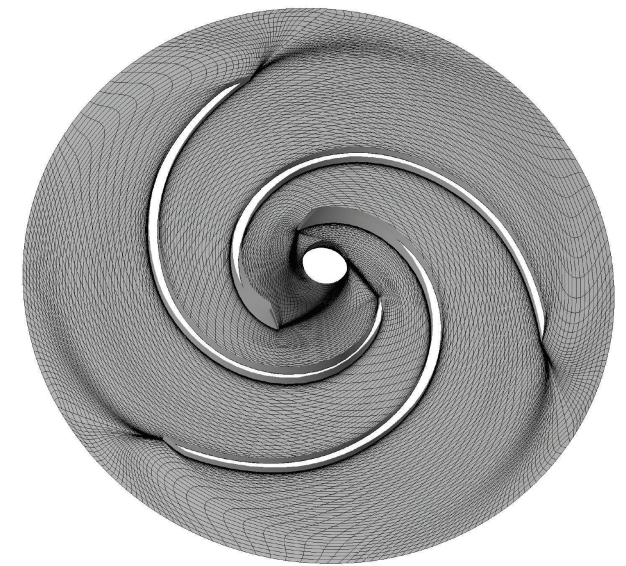

FIgURE 4: 3D view of the impeller grid.

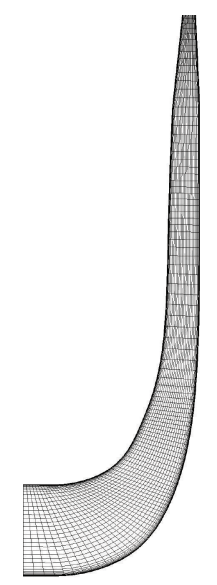

Figure 5: 2D view of the meridional channel grid.

developed at the University of Florence for compressible turbomachinery flows, was used in the present work. The concept of artificial compressibility of Chorin [13] is used to handle incompressible fluids by a time-marching approach. The time derivative of pressure, weighted by an artificial compressibility coefficient, is added to the continuity equation in order to obtain an unsteady formulation for the three-dimensional Reynolds-Averaged NavierStokes (RANS) equations written in conservative form in a curvilinear, body-fitted coordinate system.

The spatial discretization of the equations is based on a finite-volume cell-centered scheme. A blended secondand fourth-order artificial dissipation model, together with an eigenvalue scaling, is used in order to minimize the amount of artificial diffusion inside the shear layers. The equations are advanced in time using an explicit fourstage Runge-Kutta scheme, until the steady state solution is reached. In order to reduce the computational cost and speed up convergence to the steady solution, four computational techniques are employed: local time stepping, implicit residual smoothing, multigrid full-approximation storage 
(FAS), and grid refinement. Several turbulent closures are available, namely, the algebraic Baldwin-Lomax model [14], the one-equation Spalart-Allmaras model [15], and the twoequation Wilcox k- $\omega$ model $[16,17]$. Boundary conditions are imposed on solid walls (no slip), on periodic boundaries, and at inlet (total pressure and flow angles) and outlet (static pressure at hub) of the computational domain. The flow was pressurized to some extent with respect to physical pressure at the impeller inlet, in order to prevent cavitation from arising at the impeller mouth, which would make it impossible to perform computations with a single-phase fluid model (e.g., Arnone et al. [18]).

The code has recently been used for the design and optimization of centrifugal and mixed-flow pump components (Arnone et al. [19], Bonaiuti et al. [9], Boncinelli et al. [20]).

2.4. Postprocessing. Stage performance is evaluated considering fluid dynamic area-averaged quantities at two sections, the first one just upstream of the impeller leading edge and the second one downstream of the impeller trailing edge. In Figure 2, the position of the two sections is illustrated, together with a meridional view. The main analyzed quantities are as follows.

(i) Pressure Distribution. Blade pressure on both the pressure and the suction sides is reported as a function of dimensionless curvilinear abscissa $\mathrm{m} / \mathrm{m}_{\text {tot }}$, where $m_{\text {tot }}$ is calculated on the pressure and suction sides, respectively.

(ii) Spanwise Distribution. Spanwise distributions of pitch-averaged total pressure, static pressure, and absolute and relative velocities, and absolute and relative flow angles are evaluated.

(iii) Integral Quantities. Total head, flow rate, static pressure, total-to-total efficiency, outlet area-averaged meridional, and tangential velocities are computed.

(iv) Blade-to-Blade and Meridional Channel Views. Bladeto-blade and meridional channel views are used to analyze secondary flows.

(v) Suction Capability. A numerical model to solve cavitating flows directly would need a two-phase fluid model, moreover the phenomenon is typically unsteady and nonaxisymmetric. These requirements are still not suitable for everyday industrial design applications due to their high computational cost. Even if details of cavitation development cannot be provided, 3D computations can still be used as a design tool, and a cavitation inception criterion is conventionally defined in order to compare suction capabilities of different geometries. After each computation, pressure was artificially decreased until $5 \%$ of the impeller blade surface had a static pressure lower than the local vapor pressure $\left(p_{v}\right)$. The corresponding inlet total pressure $\left(p_{t, 5 \%}\right)$ was used to compute a numerical $N P S H_{R}$ of the pump at that operating condition (e.g., Bonaiuti et al. [9]).

In order to evaluate the pump specific speed, it is necessary to model all the pump components. Two approaches

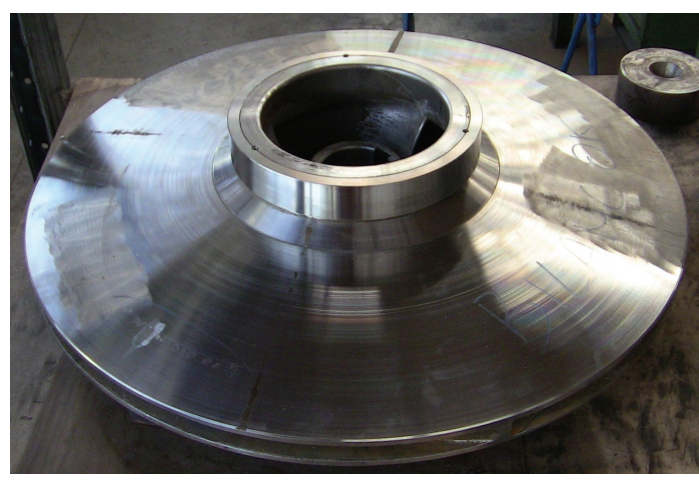

Figure 6: The $N_{s}=10.9$ pump.

can be followed: the first one is to include all the pump components in the CFD domain, and the second one consists of the CFD analysis of the impeller alone and uses a correlative approach to account for the losses of the other components. The first method has a high computational cost, and thus it is not suitable for optimization purposes. The second one is much less time consuming and more appropriate for use in an optimization procedure. For this reason, the latter approach was selected in this work for calculating the pump performance. Additional losses related to the static components, at a given flow rate, were estimated by means of a 1D code:

(i) leakages (Stepanoff [21]), windage (Gülich [22]),

(ii) ducts: inlet and outlet conical diffusers, discharge bent duct (Idel'cik [23]),

(iii) circular volute (Van den Braembussche [24]).

2.5. Artificial Neural Networks. The optimization method is based on the use of Artificial Neural Networks (ANNs). ANNs are used for the construction of metamodels of each constraint or objective function within an optimization. They are chosen mainly for one reason: the use of metamodel allows performing calculations in parallel, with a consequent reduction of the overall timescale of the activity [25]. Feedforward networks with two hidden layers are used. As far as training is concerned, a gradient-based back-propagation method is employed. In order to improve the generalization ability, a hybrid network made by multiple trained neural networks is used (e.g., [26, 27]). Effective hybridization can be accomplished by choosing different architectures, different training sets, and different initializations of weight vectors during the training process. The generalization ability of the network is evaluated by computing the prediction error over an independent validation data set.

\section{3. $N_{s}=10.9$ Pump Characteristics}

Experimental data are available for a pump with $N_{s}=10.9$, composed of an inlet conical diffuser, a shrouded centrifugal impeller with three blades (Figure 6), a circular volute, an outlet cone-shaped diffuser, and a bent duct. The main objective of this work is to design an impeller which can be 


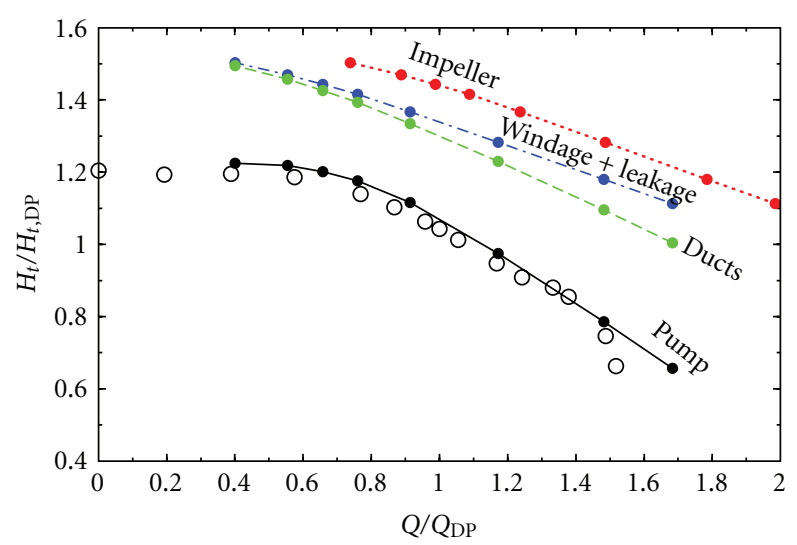

(a)

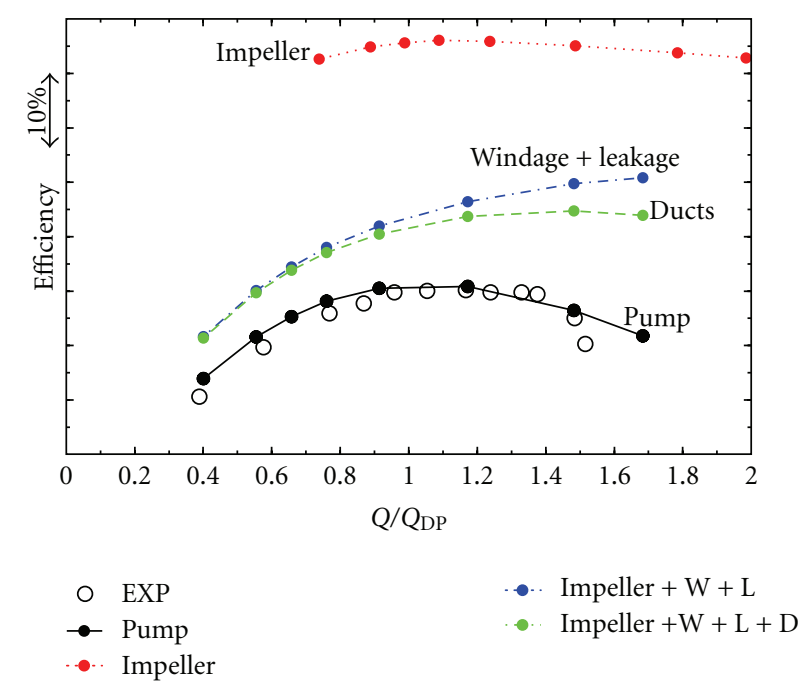

(b)

Figure 7: Comparison between predicted and measured pump characteristics $\left(N_{s}=10.9\right)$.

coupled with the same static components of the $N_{s}=10.9$ pump, in order to obtain a new pump with a $N_{s}=9.5$. With this in mind, the performance prediction capability of the proposed approach was assessed by comparing the computed and the measured characteristics of the pump with $N_{s}=10.9$ in Figure 7. The experimental data consisted of efficiency and total head versus flow rate. The flow rate range spanned from the shut-off condition to $150 \%$ of the design flow rate. The performance of the impeller was calculated with the 3D CFD solver, then the losses of the various static components, estimated with the $1 \mathrm{D}$ code, were added to give the pump characteristics. As can be seen in Figure 7, it is essential to account for all the contributions in order to obtain a reliable prediction of the pump performance.

The agreement is fairly good with the exception of the higher flow rates where a steep degradation is visible in the measured pump performance due to cavitation. Going towards the low flow rates, unsteady flow phenomena tend to arise, and it is not possible to calculate the impeller with a steady-state approach.
TAble 1: Degrees of freedom and their influence on pump's performance.

\begin{tabular}{lccccccccccc}
\hline & $b_{2}$ & $\beta_{2}$ & $\beta_{1}$ & $\beta_{m}$ & $m_{m}$ & $r_{\mathrm{LE}}$ & $\gamma_{\mathrm{LE}}$ & $x_{4 H}$ & $x_{4 T}$ & $r_{5 H}$ & $r_{5 T}$ \\
\hline$H_{t} ; N_{s}$ & $\checkmark$ & $\checkmark$ & & $\checkmark$ & $\checkmark$ & & & & & & \\
$N_{P S H_{R}}$ & & $\checkmark$ & $\checkmark$ & $\checkmark$ & $\checkmark$ & $\checkmark$ & $\checkmark$ & $\checkmark$ & $\checkmark$ & $\checkmark$ & $\checkmark$ \\
$\mathrm{BEP}$ at DP & $\checkmark$ & $\checkmark$ & $\checkmark$ & & & & & & & & \\
$\eta_{t t}$ & $\checkmark$ & $\checkmark$ & $\checkmark$ & $\checkmark$ & $\checkmark$ & $\checkmark$ & $\checkmark$ & $\checkmark$ & $\checkmark$ & $\checkmark$ & $\checkmark$ \\
\hline
\end{tabular}

\section{Design and Optimization}

The objectives of the design of the new pump were

(i) specific-speed number of $N_{s}=9.5$ at the design flow rate (DP) with a prescribed rotational speed,

(ii) overall minimum value of total-to-total efficiency of the pump $\left(\eta_{t t \text {,target }}\right)$,

(iii) good suction capability $\left(\mathrm{NPSH}_{R}\right)$.

The imposed constraints follow the industrial requirements of feasibility. In the low specific speed range, the impeller passage height at the outlet $\left(b_{2}\right)$ is a key parameter, since in the conventional design procedures it would usually be too narrow to be manufactured. For this reason the impeller should respect a minimum outlet width $\left(b_{2}\right)$, a maximum azimuthal blade angle $\theta$, and a minimum blade thickness. A 2D-blade profile in the $(r, \theta)$ plane is needed in order to ensure an easily demoulding impeller. The impeller encumbrance needs to be the same as the $N_{s}=10.9$ pump so that it can be coupled with the same inlet duct and the same volute.

The chosen degrees of freedom are 11 geometrical parameters of the impeller. This choice has been carried out based on the specified target to be reached (see Table 1). Sobol's quasi-random sequence of training data is used in order to ensure a better coverage over the design space and minimize the number of CFD computations. The ANNs are trained using an off-line procedure and generate a response surface. In the present application, the validation sample set is a random subset of the CFD calculations performed on the basis of Sobol's sequence; this subset is not included in the training set. A Monte Carlo method is then used to select a set of optimum candidates which are verified with CFD calculations thus allowing the designer to choose the optimum solution.

Since the industrial requirement is to design a $2 \mathrm{D}$ blade in the $(r, \theta)$ plane, the hub $\beta_{b}$ distribution only has been modeled with a three-control-point Bèzier curve (see Figure 3 ). The first point is the LE blade angle $\beta_{1}$, the last point is the TE blade angle $\beta_{2}$, and the second one is located at an intermediate meridional coordinate of the blade $m_{m}$. This simple type of distribution allows one not to have any inflection point, furthermore, with pumps characterized by a strong radial development, the blade angle curve is not so effective in terms of pump performance. Hub and shroud meridional contours are shaped with a third-degree B-spline curve with 8 control points (Figure 2). 
Both the abscissa and ordinate of each control point are adimensionalized with respect to the axial and radial length of the meridional channel, respectively. This choice has been pursued in order to easily shape the meridional contours when the other geometrical parameters, like $b_{2}$, are modified in the optimization procedure (e.g., Casey et al. [8]). Only the abscissa of the fourth control point and the ordinate of the fifth control point, at both hub and shroud, were modified because of their influence on the front part of the blade loading and on the incidence.

Blade sections, stacked at the TE, have a constant thickness over the whole camber line, the leading edge is shaped with a prescribed curve, and the trailing edge is cut at a constant radius.

In the present application, the number of blades is not a degree of freedom and only two configurations have been investigated for industrial needs: a 3-blade impeller and a 5-blade impeller. A 5-blade impeller configuration, with the same constraints on the minimum value of $b_{2}$ and maximum value of azimuthal angle $\theta$ development, reduces the probability of recirculated flow both in the blade passage and at the impeller outlet, in spite of a reduction in the suction capability.

Hence, starting from the same set of parameters, two optimizations were carried out at the design point (DP), and two different strategies were chosen. For the 3-blade impeller, the optimized geometry was obtained by means of a multistep analysis. During each step, a limited number of parameters were varied, and the range of variation was progressively refined. For the 5-blade impeller, a one-step global method was adopted, and all the parameters were involved in the optimization procedure with a large range of variation. Generally speaking, the computational cost of the first method is lower, but the results can be affected by the designer's choice of the various subset of parameters considered in each single step. On the contrary, the main advantage of the global method is that the only critical choice is related to the initial selection of the parameters. The influence of all the parameters is then investigated at the same time. The higher the number of parameters, the higher the number of computations needed for an appropriate training of the neural network. As a result, the second method would require a much higher number of calculations with respect to the first one. Despite this fact, the two optimization procedures were applied by using about the same amount of computational resources, for testing them against a tight industrial design scheduling. Each computed geometry required about 1.5 hours on a Xeon CPU X5650 at $2.67 \mathrm{GHz}$, so that a single optimization was obtained in 3 days on a 40 -core cluster.

4.1. 3-Blade Impeller Optimization. A three-step method was chosen to perform this optimization. Since the specific-speed number $\left(N_{s}=9.5\right)$ is of primary concern, in the first step the subset of parameters $\left(b_{2}, \beta_{m}, m_{m}, \beta_{2}\right)$ was chosen on the basis of their influence on the total head (see Table 1 ). In order to adjust the incidence for different flow rates, $\beta_{1}$ and $\gamma_{\mathrm{LE}}$ were considered as well, for a total of 6 parameters (see Table 2).
TABLE 2: Parameters varied in 3-blade impeller optimization's steps.

\begin{tabular}{lccccccccccc}
\hline OPT STEP & $b_{2}$ & $\beta_{2}$ & $\beta_{1}$ & $\beta_{m}$ & $m_{m}$ & $r_{\mathrm{LE}}$ & $\gamma_{\mathrm{LE}}$ & $x_{4 H}$ & $x_{4 T}$ & $r_{5 H}$ & $r_{5 T}$ \\
\hline 1st & $\checkmark$ & $\checkmark$ & $\checkmark$ & $\checkmark$ & $\checkmark$ & & $\checkmark$ & & & & \\
2nd & $\checkmark$ & & $\checkmark$ & & & $\checkmark$ & $\checkmark$ & $\checkmark$ & $\checkmark$ & $\checkmark$ & $\checkmark$ \\
3rd & $\checkmark$ & $\checkmark$ & $\checkmark$ & $\checkmark$ & $\checkmark$ & & $\checkmark$ & & & & \\
\hline
\end{tabular}

TABLE 3: Sample and validation set sizes for the two metamodels.

\begin{tabular}{lcc}
\hline & sample set size & validation set size \\
\hline OPT3 & $676 / 594 / 150$ & $20 / 30 / 30$ \\
OPT5 & 1757 & 30 \\
\hline
\end{tabular}

The ranges of variation chosen were as big as possible with respect to the industrial feasibility.

Once the $N_{s}$ was targeted, the next step involved the research of the maximum efficiency and an increased suction capability $\left(\mathrm{NPSH}_{R}\right)$. The 8 parameters involved are the angle $\gamma_{\mathrm{LE}}$, the meridional channel contours $\left(x_{4 H}, x_{4 T}, r_{5 H}, r_{5 T}\right)$, the LE radius $r_{\mathrm{LE}}$, the outlet impeller width $b_{2}$, and the blade inlet angle $\beta_{1}$. The parameter $b_{2}$ has a strong influence on the volute, and hence on the pump performance. For this reason, it was included in the subset considered for the second step with a narrow range of variation.

The final step of the optimization involved the 6 parameters, yet investigated in the first step, with a refined range of variation. In fact, this subset of parameters is the most effective for obtaining the target specific speed. Results of the computations performed in the various steps are summarized in Figure 8(a), where the pump efficiency is plotted against the specific speed. Even if three different training sets are shown in Figure 8(a), a unique metamodel was used. After each step, the same ANN was actually retrained using an extra sample set. The sample set size was 676,594 , and 150 for the three steps, respectively (for a total of 1420 samples, see Table 3 ).

4.2. 5-Blade Impeller Optimization. In this case, the optimization was carried out in a single step, and the eleven parameters were allowed to vary at the same time, covering a range of values as wide as possible. The $N_{s}$, the efficiency, and the suction capability were all pursued at the same time. The optimization cloud, composed of 1757 samples (see Table 3), is shown in Figure 8(b), the optimum geometries typically describe a Pareto front.

4.3. Results. Two-response surfaces were generated for the 3 blade and 5-blade impeller, respectively. Hence, two different Artificial Neural Networks were trained since the goal was to obtain two optimized impellers with a different number of blades. The two optimization clouds are shown in Figures $8(\mathrm{a})$ and $8(\mathrm{~b})$, where the efficiency is plotted against the specific speed. In these figures the optimized geometries eventually suggested by the neural networks are highlighted. These two geometries, called OPT3 and OPT5, respectively, were calculated with $\mathrm{CFD}$ and postprocessed with the 


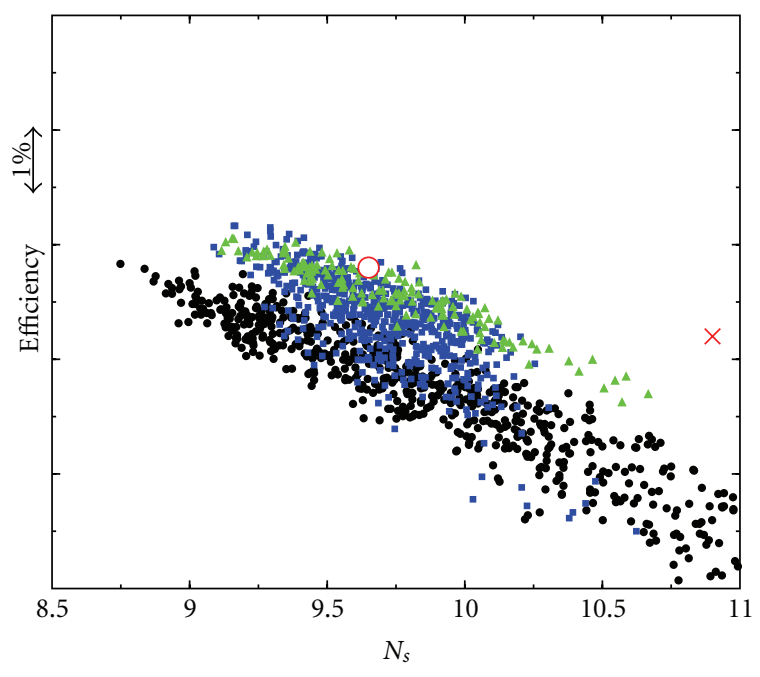

- Training set-1st step

- Training set-2nd step

$\Delta$ Training set-3rd step

(a)

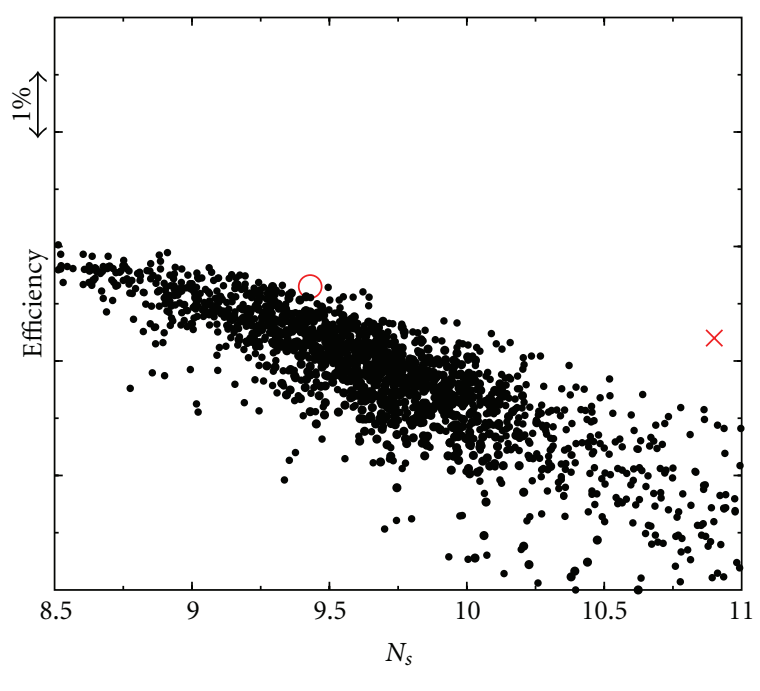

- Training set

OPT5

$\times N_{s}=10.9$ pump

(b)

FIGURE 8: The optimization clouds: (a) 3-blade impeller, (b) 5-blade impeller.

correlation-based 1D tool. The performance predicted by the neural networks are compared with the ones obtained from the computations in Table 4 . As can be seen, the neural network predictions are in good agreement with the simulations.

The predicted operating characteristics of the two optimized geometries are compared with experimental data in Figure 9, where the computed flow rate goes from $20 \%$ to the $170 \%$ of the DP. The agreement with measurements is seen to be very good for both the 3 - and the 5-blade cases, in

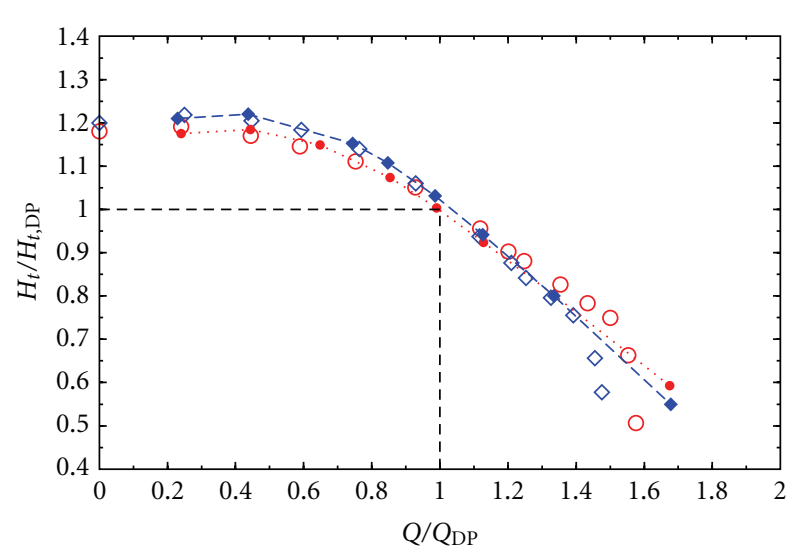

(a)

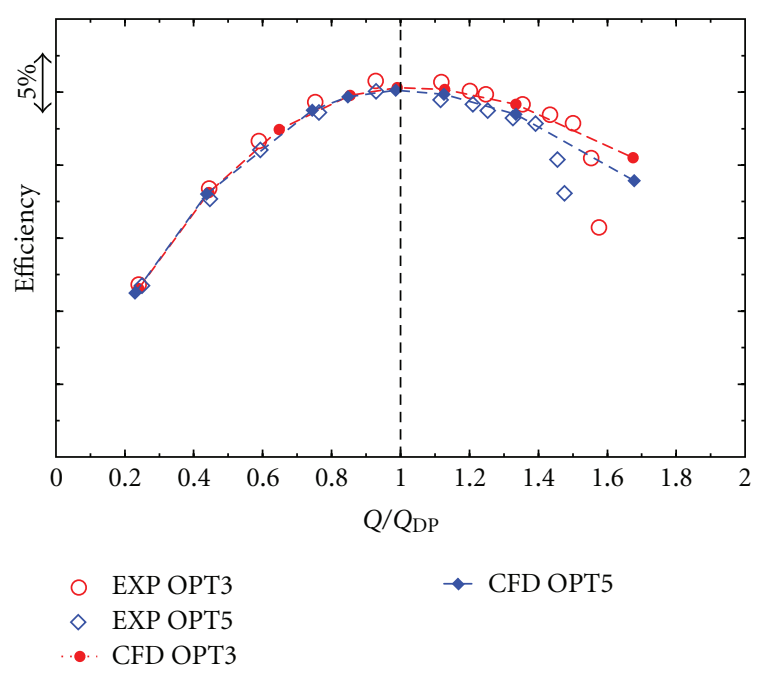

(b)

Figure 9: Comparison between computed 3-blade and 5-blade pump characteristics $\left(N_{s}=9.5\right)$.

TABle 4: Comparison between pump performance predicted by ANN and calculated with CFD.

\begin{tabular}{lcccc}
\hline & \multicolumn{2}{c}{$N_{s}$} & \multicolumn{2}{c}{$\eta_{\mathrm{tt}}-\eta_{\mathrm{tt} \text { target }}$} \\
& ANN & CFD & ANN & CFD \\
\hline OPT3 & 9.59 & 9.65 & $+0.67 \%$ & $+0.60 \%$ \\
OPT5 & 9.56 & 9.43 & $+0.24 \%$ & $+0.27 \%$ \\
\hline
\end{tabular}

particular the higher level of head obtained with the 5-blade impeller is correctly reproduced. An exception is again found for flow rates higher than $140 \% Q_{D P}$, where experimental data show the typical performance degradation due to the cavitation of the pump. It can be noticed how the drop in the measured characteristics starts at different flow rates for the 3-blade and the 5-blade impellers. This is caused by a different NPSH available at the suction of the pump for the two cases. As noticed before, the same behaviour cannot be obtained with the calculation based on a single-phase fluid model. Since the optimizations were performed for the 


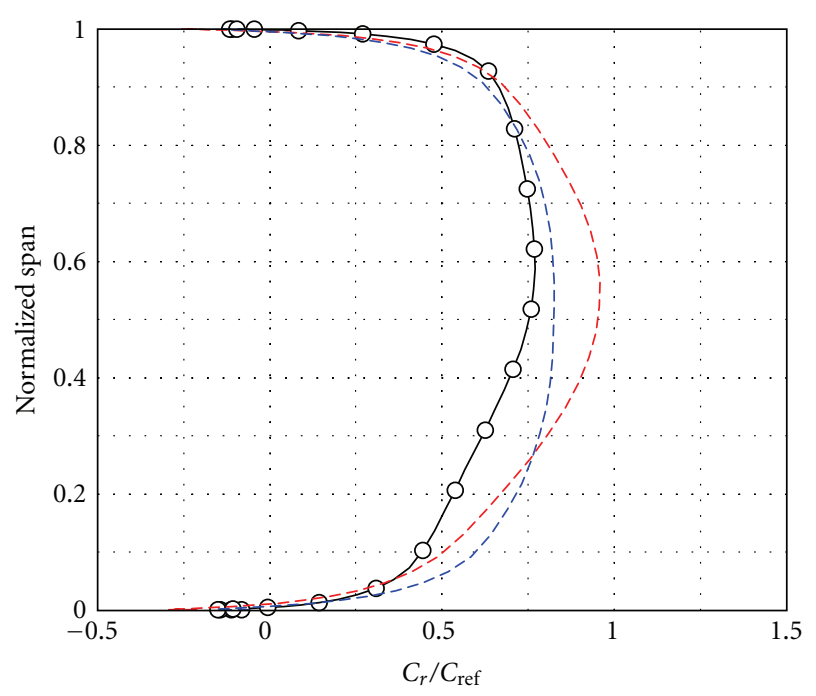

(a)

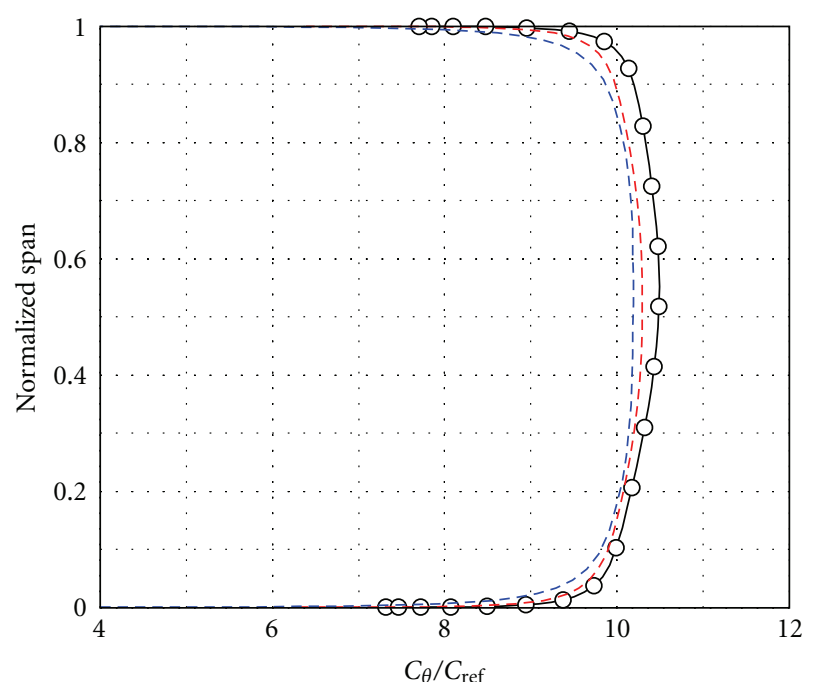

(b)

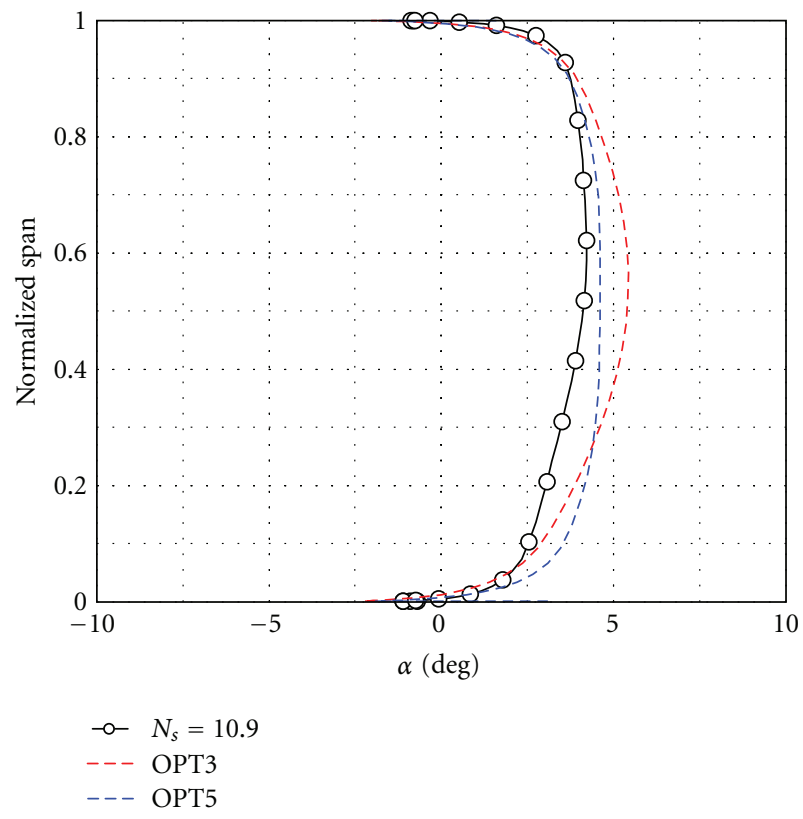

(c)

FIGURE 10: Spanwise distributions at DP in the outlet region.

design point operating condition, and the maximization of the efficiency is one of the target, it can be expected that the design point will match the best efficiency point. This fact can be verified from the predicted and measured operating characteristics.

Figure 10 shows the spanwise distributions of areaaveraged flow quantities at the impeller outlet section. Both the 3-blade and the 5-blade optimized impellers have a value of the outlet width $b_{2}$ close to the minimum value fixed as a constraint. This is because a low value of $b_{2}$ tends to reduce the possibility of recirculating flow regions inside the impeller passage. Since the impeller for the $N_{s}=9.5$ pump should be coupled with the same volute of the $N_{s}=10.9$ pump, results obtained at the impeller exit are compared for the three geometries. The optimized impellers present a higher level of radial velocity at the blade exit; the velocity profile obtained with the 5-blade configuration is more uniform along the span with a lower predisposition to separation. The circumferential velocity level is substantially unaltered for the optimized geometries with respect to the $N_{s}=$ 10.9 pump, and the absolute flow angle is higher of less than $1^{\circ}$. This should not affect the volute performance too much.

The relative velocity magnitude contours in a blade-toblade section at midspan are shown in Figure 11 for the 


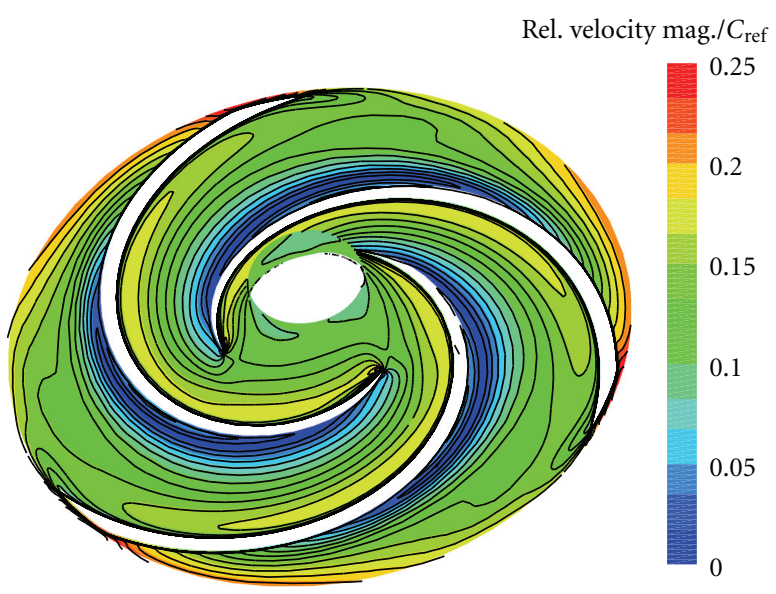

(a) OPT3

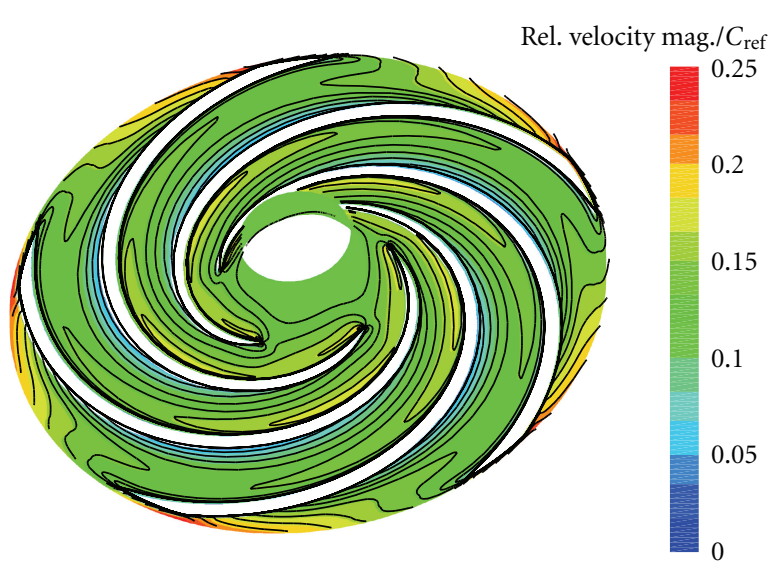

(b) OPT5

FIgure 11: 3D view of the impeller flow field at DP $\left(N_{s}=9.5\right)$.

two optimized configurations at the design point. The 3blade impeller presents a large region of recirculated flow on the pressure side originating just downstream the blade leading edge (Figure 11(a)). Due to the limit imposed on the azimuthal angle development of the blade, it was not possible to avoid this recirculation. The recirculation is suppressed by adopting a higher number of blades, so that the constraint on blade angular development issue is mitigated (Figure 11(b)). In Figure 12, nondimensional static pressure distributions on the impeller blade are reported as a function of the relative curvilinear abscissa at 10\%, 50\%, and $90 \%$ of the blade span. Since the pump inlet duct should be maintained, the suction capabilities of the $N_{s}=10.9$ and $N_{s}=9.5$ pumps are compared. The static pressure values shown in these figures are obtained after subtracting the added level of pressurization needed to perform computations while preventing cavitation from arising. The value of zero pressure has been marked with a dash-dot line for reference. This allowed the designer to find out which portions of the blade were likely to be affected by cavitation by looking for negative values of static pressure, neglecting vapor pressure compared with inlet total pressure (e.g., Arnone et al. [18], Bonaiuti

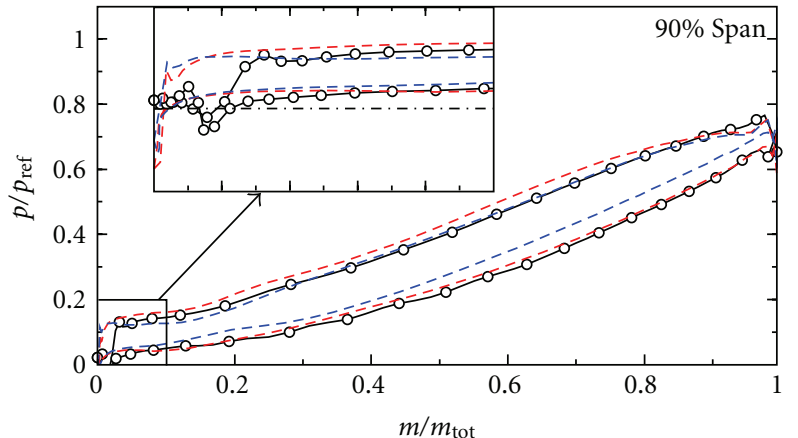

(a)

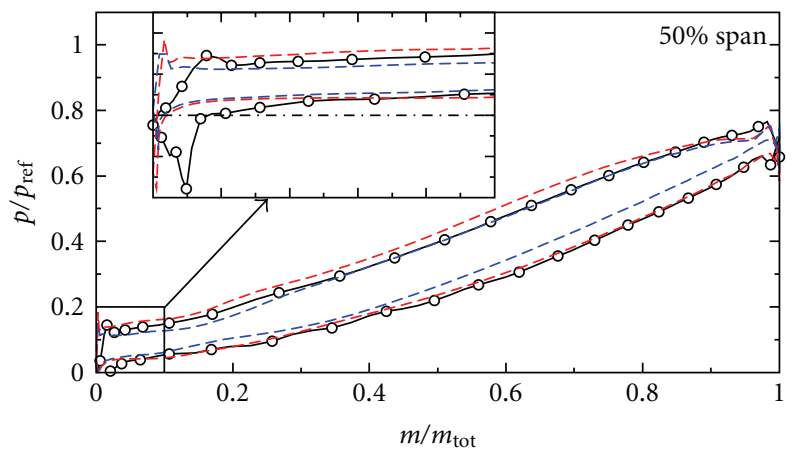

(b)

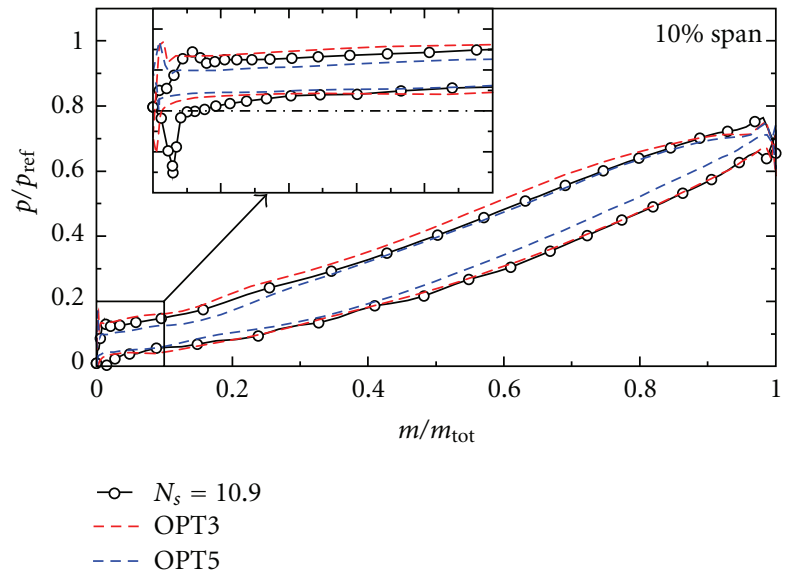

(c)

FIgure 12: Pressure distributions at BEP for three different spans, $N_{s}=9.5$ (OPT3-OPT5) and $N_{s}=10.9$ pumps.

et al. [9]). The pressure distributions of the two optimized impellers present a higher level of pressure on both the suction and the pressure side with respect to the $N_{s}=10.9$ geometry from hub to tip. These blade load distributions were obtained with a low value of the flow incidence (i.e., about $2-3^{\circ}$ ). This kind of pressure distributions in the forepart of the blade should ensure a better suction performance together with a low risk of cavitation inception $\left(\mathrm{NPSH}_{\mathrm{R}}\right)$. 


\section{Conclusions}

In this paper, the aerodynamic design of the impeller of a low specific-speed pump was presented and described in detail. The design targets were to match the design point and the best efficiency point with a prescribed specific-speed number $\left(N_{s}=9.5\right)$ together with a guaranteed value of the pump efficiency and a good suction capability $\left(\mathrm{NPSH}_{R}\right)$. The main goal was to accomplish these targets under defined geometrical constraints which reflected the industrial needs of feasibility and low production costs. For these reasons, the new impeller needs to be coupled with the same static components of an already existing pump with a slightly higher specific-speed number of $N_{s}=10.9$.

Such a process started with the CFD analysis of the $N_{s}=10.9$ pump impeller configuration. Static components of the pump, like the diffuser ducts and the volute, as well as additional loss contributes due to leakages and windage, were obtained by means of a 1D-prediction tool based on widely used correlations. The performance capability prediction of the model was assessed by comparing the pump's computed characteristics with the measurements, and a fairly good agreement was found on the whole operating range.

The aerodynamic design of the impeller was carried out using a parameterization tool which allowed a direct control on manufacturing and structural constraints and made it possible to identify the effect of single parameters on impeller aerodynamic performance. The overall design procedure exploited a Neural-Network-based approach which was a fast and powerful tool for the optimization of the pump performance. Two configurations with a 3-blade (OPT3) and a 5-blade (OPT5) impellers were selected and optimized with the additional target of obtaining a good suction capability $\left(\mathrm{NPSH}_{R}\right)$.

Both configurations satisfied the desired targets of specific speed and efficiency, with an increase of about $0.6 \%$, for OPT3, and $0.3 \%$, for OPT5, with respect to the minimum required value at $\mathrm{DP}$. The 5 -blade impeller configuration was able to mitigate the flow recirculation inside the impeller passages which instead tended to persist with the 3-blade solution, due to the constraints on the impeller exit width and on the blade angular development. Results obtained with the proposed optimization method were assessed against experimental data for the pumps with the 3-blade and the 5-blade impellers. A very good agreement was found between the predicted and measured operating characteristics demonstrating how the proposed approach offers an economical and reliable design tool for industrial needs.

\section{Nomenclature}

$b$ : Impeller width (m)

$c$ : Absolute velocity magnitude $(\mathrm{m} / \mathrm{s})$

$g$ : Gravity acceleration $\left(\mathrm{m} / \mathrm{s}^{2}\right)$

$H$ : Head (m)

$m$ : Meridional abscissa $(\mathrm{m})$

$n$ : Impeller rotational speed (rpm)

$N_{s}$ : Specific-speed number, $n Q^{1 / 2} H^{-3 / 4}\left(\mathrm{rpm} \mathrm{m} m^{m / 4} s^{-1 / 2}\right)$

$P$ : Pressure $(\mathrm{Pa})$ $p_{t ; 5 \%}$ : Inlet total pressure for cavitation criterion $(\mathrm{Pa})$

Q Volume flow rate $(\mathrm{m} 3 / \mathrm{s})$

$x ; r ; \theta$ : Cylindrical coordinates.

Greek

$\alpha$ : Flow angle, $\tan ^{-1}\left(c_{\theta} / c_{m}\right)\left(^{\circ}\right)$

$\beta$ : Metal angle $\left(^{\circ}\right)$

$\eta$ : Efficiency, $\rho g Q H / P$

$\gamma$ : Slope angle in the meridional plane $\left(^{\circ}\right)$

$\rho$ : Density $\left(\mathrm{kg} / \mathrm{m}^{3}\right)$

$\theta$ : Azimuthal angle $\left(^{\circ}\right)$.

\section{Subscripts}

1: Inlet section

2: $\quad$ Outlet section

$\theta$ : Circumferential

$b$ : $\quad$ Blade

$H$ : Hub

$m$ : Intermediate, meridional

max: Maximum value

$r: \quad$ Radial

ref: Reference value

T: Tip

$t$ : Total

$v$ : Vapor

$x$ : Axial.

\section{Acronyms}

ANN: Artificial Neural Networks

BEP: Best efficiency point

CFD: Computational fluid dynamics

DP: Design point

LE: $\quad$ Leading edge

$N_{P S H}$ : Net positive suction head, $\left(p_{t ; 5 \%}-p_{v}\right) / \rho g$

OPT3: Optimized 3-blade impeller

OPT5: Optimized 5-blade impeller

TE: $\quad$ Trailing edge.

\section{Acknowledgments}

The authors would like to express their appreciation for the financial support from the Italian Ministero dell'Istruzione, Universitá e Ricerca (MIUR), under the Research Project PACOMAR no. DM29847. They would also like to express their gratitude to Ing. Andrea Schneider of the University of Florence for the numerous and fruitful discussions on the use of artificial neural networks.

\section{References}

[1] S. Gopalakrishnan, "Pump research and development: past, present, and future- an American perspective," Journal of Fluids Engineering, Transactions of the ASME, vol. 121, no. 2, pp. 237-247, 1999. 
[2] S. Pierret and R. A. Van Den Braembussche, "Turbomachinery blade design using a Navier-Stokes solver and Artificial Neural Network," Journal of Turbomachinery, vol. 121, no. 2, pp. 326332, 1999.

[3] K. Ashihara and A. Goto, "Turbomachinery blade design Using 3-D inverse design method, CFD and optimization algorithms," ASME Paper 2001-GT-0358, 2001.

[4] A. Goto, M. Nohmi, T. Sakurai, and Y. Sogawa, "Hydrodynamic design system for pumps based on 3-D CAD, CFD, and inverse design method," Journal of Fluids Engineering, Transactions of the ASME, vol. 124, no. 2, pp. 329-335, 2002.

[5] S. Pierret, R. Filomeno Coelho, and H. Kato, "Multidisciplinary and multiple operating points shape optimization of three-dimensional compressor blades," Structural and Multidisciplinary Optimization, vol. 33, no. 1, pp. 61-70, 2007.

[6] D. Bonaiuti, A. Arnone, M. Ermini, and L. Baldassarre, "Analysis and optimization of transonic centrifugal compressor impellers using the design of experiments technique," Journal of Turbomachinery, vol. 128, no. 4, pp. 786-797, 2006.

[7] D. Bonaiuti and M. Zangeneh, "On the coupling of inverse design and optimization techniques for the multiobjective, multipoint design of turbomachinery blades," Journal of Turbomachinery, vol. 131, no. 2, p. 021014, 2009.

[8] M. Casey, F. Gersbach, and C. Robinson, "An optimization technique for radial compressors impellers," ASME Paper GT2008-50561, 2008.

[9] D. Bonaiuti, A. Arnone, U. Corradini, and M. Bernacca, "Aerodynamic redesign of a mixed-flow pump stage," ASME Paper 2003-3506, 2003.

[10] A. Arnone, "Viscous analysis of three-dimensional rotor flow using a multigrid method," Journal of Turbomachinery, vol. 116, no. 3, pp. 435-445, 1994.

[11] A. Arnone, "Multigrid methods for turbomachinery NavierStokes calculations," in Solution Techniques for Large-Scale CFD Problems, W. G. Habashi, Ed., John Wiley \& Sons, 1995.

[12] A. Arnone and R. Pacciani, "Three-dimensional viscous analysis of centrifugal impellers using the incompressible Navier-Stokes equations," in Proceedings of the 1st European Conference on Turbomachinery, pp. 181-195, Erlangen, Germany, 1995.

[13] A. J. Chorin, "A numerical method for solving incompressible viscous flow problems," Journal of Computational Physics, vol. 2, no. 1, pp. 12-26, 1967.

[14] B. S. Baldwin and H. Lomax, "Thin layer approximation and algebraic model for separated turbulent flows," AIAA Paper 78-257, 1978

[15] P. R. Spalart and S. R. Allmaras, "One-equation turbulence model for aerodynamic flows," La Recherche Aérospatiale, no. 1, pp. 5-21, 1994.

[16] D. C. Wilcox, Turbulence Modeling for CFD, DCW, La Cañada, Calif, USA, 2nd edition, 1998.

[17] D. C. Wilcox, "Formulation of the $\mathrm{k}-\omega$ turbulence model revisited," AIAA Journal, vol. 46, no. 11, pp. 2823-2838, 2008.

[18] A. Arnone, P. Boncinelli, A. Capuani, E. Spano, and C. Rebattet, "Ariane 5 TPLOX inducer design Strategies to enhance cavitating performance," in Proceedings of the 4th International Symposium on Cavitation (CAV '01), Pasadena, Calif, USA, June 2001.

[19] A. Arnone, P. Boncinelli, A. Munari, and E. Spano, "Application of CFD Techniques to the Design of the Ariane 5 Turbopump," AIAA Journal, vol. 46, no. 11, pp. 2823-2838, 2008.
[20] P. Boncinelli, R. Biagi, A. Focacci et al., "Bowl-type diffusers for low specific-speed pumps: an industrial application," Journal of Turbomachinery, vol. 130, no. 3, p. 031013, 2008.

[21] A. J. Stepanoff, Centrifugal and Axial Flow Pumps, Krieger Publishing Company, 2nd edition, 1957.

[22] J. F. Gülich, Centrifugal Pumps, Springer, Berlin, Germany, 1st edition, 2008.

[23] I. E. Idel'cik, Mémento des Pertes de Charge, Edition Eyrolles, 3rd edition, 1986.

[24] R. A. Van den Braembussche, "Flow and loss mechanisms in volutes of centrifugal pumps," NATO RTO-EN-AVT-143-12, 2007.

[25] A. Cichocki and R. Unbehauen, Neural Networks for Optimization and Signal Processing, John Wiley \& Sons, New York, NY, USA, 1994.

[26] M. M. Rai, "Three-dimensional aerodynamic design using artificial neural networks," AIAA Paper 2002-0987, 2002.

[27] F. Rubechini, A. Schneider, A. Arnone, S. Cecchi, and F. Malavasi, "A redesign strategy to improve the efficiency of a 17-stage steam turbine," ASME Paper GT2009-60083, 2009. 

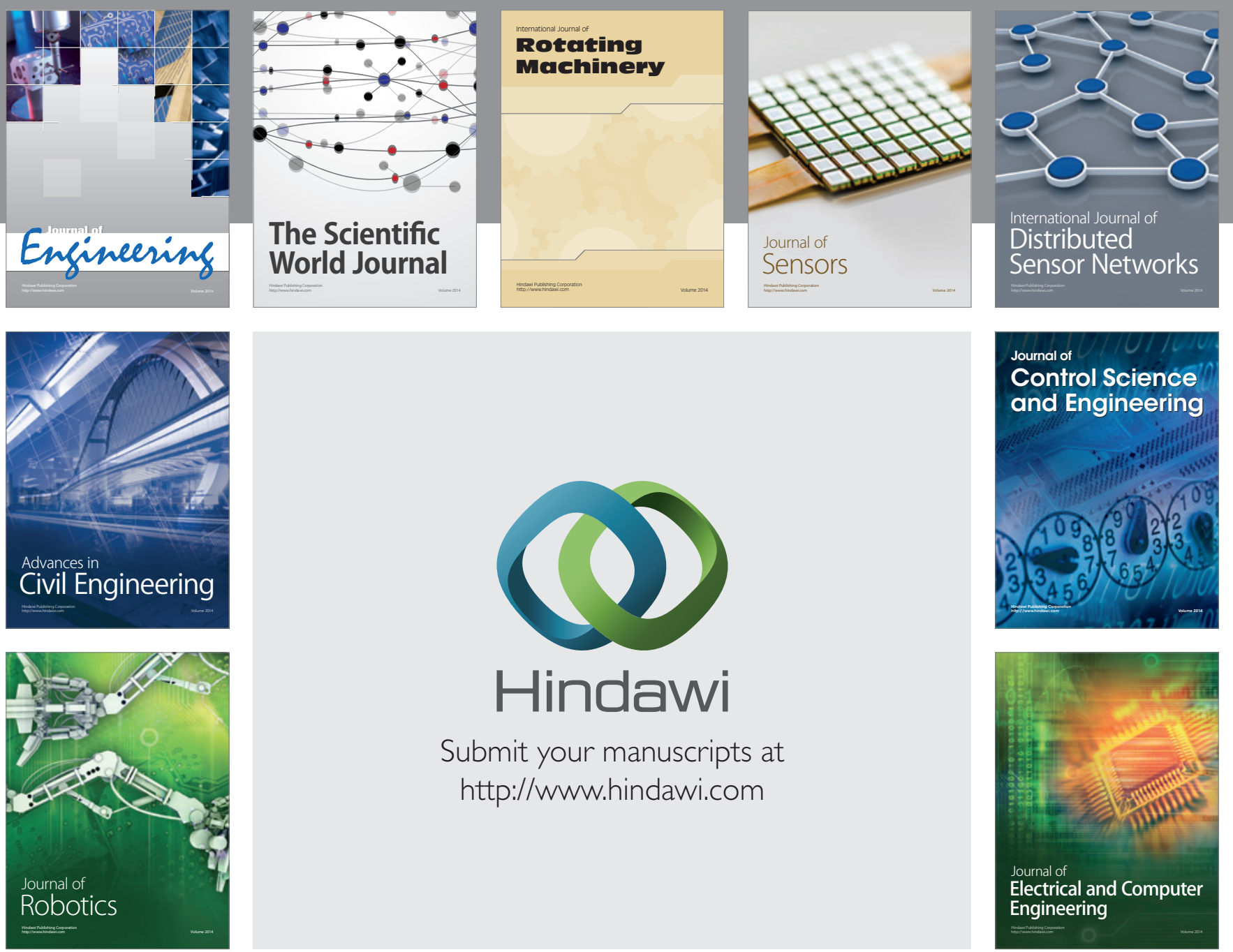

Submit your manuscripts at

http://www.hindawi.com
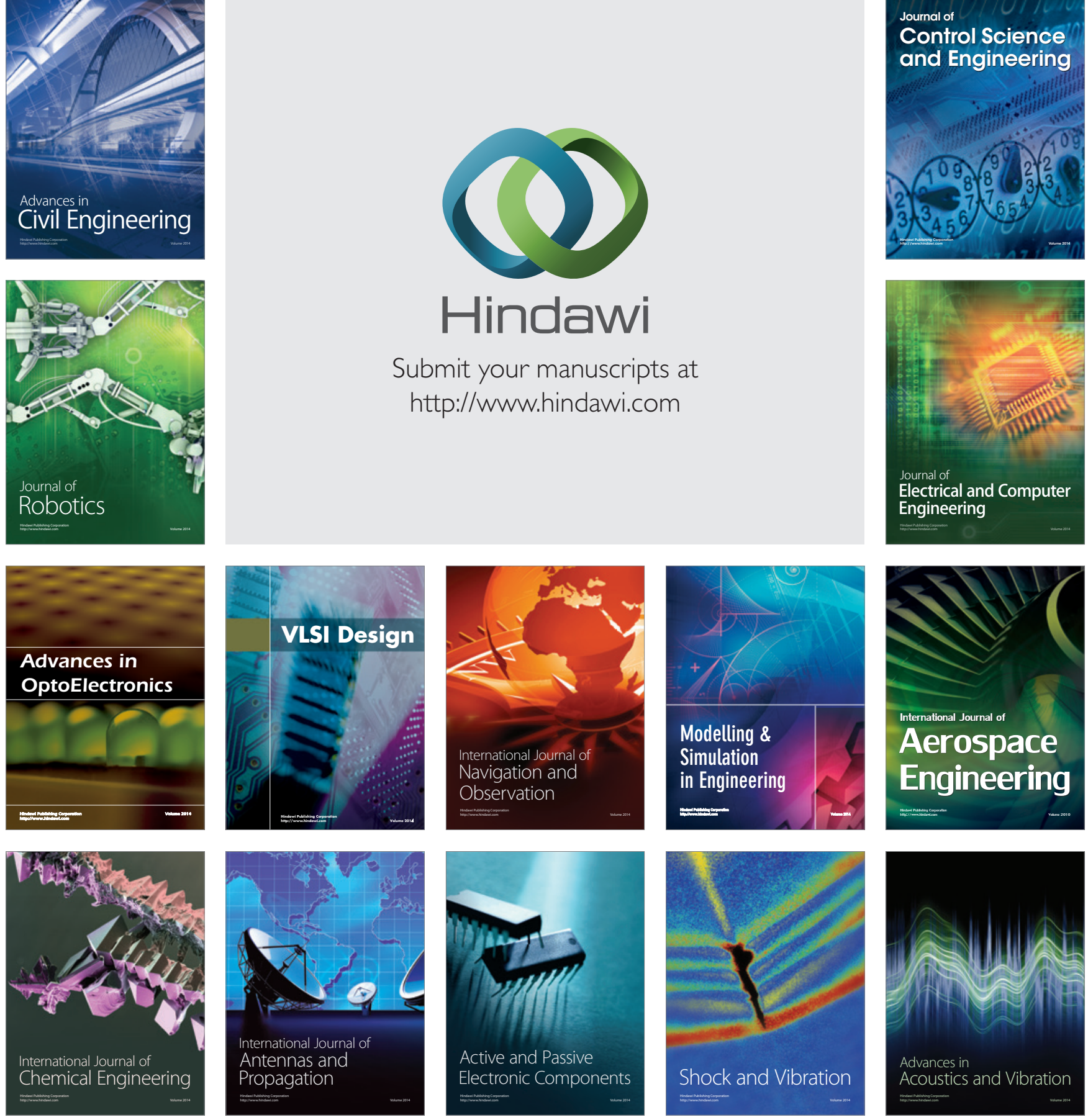